\title{
Investigating the use of terahertz pulsed time domain reflection imaging for the study of fabric layers of an Egyptian mummy
}

Kaori Fukunaga

kaori@nict.go.jp

Emilia Cortes

\section{Antonino Cosentino}

Isabel Stünkel

Marco Leona

Irl N. Duling III

David T. Mininberg
National Institute of Information and Communications Technology, Tokyo, Japan

The Metropolitan Museum of Art, New York, USA

The Metropolitan Museum of Art, New York, USA

The Metropolitan Museum of Art, New York, USA

The Metropolitan Museum of Art, New York, USA

Picometrix, LLC, Ann Arbor, USA

Weill Cornell Medical College, New York, USA

This paper reports the first use of terahertz time domain reflection imaging involving textiles on part of a complete human mummy, still in original wrapping. X-ray technique has been used extensively to investigate anatomical features, since X-ray pass through the wrapping. Terahertz waves, on the other hand, can penetrate into non-metallic materials and its reflection depends on the refractive index of materials at the interface, such as textiles and the air. The mummy of Kharushere (ca. $945-712$ B.C.) was examined by using Terahertz time domain reflection imaging in the Egyptian galleries of The Metropolitan Museum of Art. Experimental results suggest that the Terahetz imaging is a promising technique for probing the fabric layers surrounding Egyptian mummies, although it is still very limited in its current state. In the future it could become a useful complement to CT scanning when materials with low radiographic density and contrast are being investigated. [DOI: http://dx.doi.org/10.2971/jeos.2011.11040]

Keywords: terahertz, cultural heritage, nondestructive test

\section{INTRODUCTION}

Conventional X-ray radiography, and three-dimensional computerized tomography (CT), have been used extensively in order to image the internal anatomical features of Egyptian mummies and to localize objects that may have been placed underneath or within the bandages, without unwrapping the mummies. Information obtained by conventional and CT radiography has allowed scholars to gain information on the individuals lives, age and cause of death $[1,2,3]$. Contrast in radiographic images is a function of atomic number differences among the materials present in the object investigated. While careful selection of accelerating voltage and exposure conditions can significantly improve the quality of a radiograph, objects or portions of an object differing only slightly in radiographic density often cannot be distinguished. Similarly, objects having low radiographic density cannot be imaged efficiently by X-ray techniques when they are hidden behind high radiographic density objects. In terahertz pulsed time domain reflection imaging $(\mathrm{THz}$ imaging) [4,5], contrast depends on the refractive index of the materials. Thus, $\mathrm{THz}$ imaging could become a complement to $X$-ray techniques for the non-invasive investigation of ancient mummies.

\section{THZ IMAGING}

While X-rays can be used to image the internal features of the mummified body, pulsed $\mathrm{THz}$ waves can be used to probe the layers of bandages constituting the mummy wrappings. The pulsed $\mathrm{THz}$ waves are reflected by any discontinuity in the probed material: this gives $\mathrm{THz}$ imaging the potential to provide indications on the sequence of fabric layers and on eventual objects placed in-between the bandages. $\mathrm{THz}$ radiation at the levels used for imaging is not only safe for the operator, but it can also be considered completely non destructive for the objects investigated. Furthermore, THz scanners are mobile and compact: they can be transported easily and can be used in museum galleries, as well as at archaeological sites without any concern about radiation hazard. THz imaging has already drawn considerable attention as a useful nondestructive method for the analysis of the internal structure of works of art $[6,7,8,9]$, and opaque objects in general. Indeed, $\mathrm{THz}$ imaging systems have been used previously on the small mummy of a fish and on mummified human body parts [10]. In both cases the work was aimed at studying the mummified soft-tissues close to the surface.

In the present study, the National Institute of Information and Communications Technology (Tokyo, Japan) and The Metropolitan Museum of Art (New York, USA) investigated by $\mathrm{THz}$ pulsed time domain reflection imaging the mummy of 
Kharushere, dated to Dynasty 22, ca. 945-712 B.C. (Fig. 1). The mummy and its coffin set was excavated by Gaston Maspero in 1885 and purchased by the museum from the Egyptian government in 1886. Previous radiographic investigations of the mummy of Kharushere [11] showed the skeleton of an adult male with what are possibly visceral packages in the abdominal cavity and four amulets on the body. For our investigation we used a time domain reflection imaging system (T-Ray $4000 \AA$, Picometrix, Ann Arbor, MI, USA [12]) in the 0.1-1.5 $\mathrm{THz}$ frequency range. Time domain reflection imaging uses a pulsed $\mathrm{THz}$ wave as a probe propagating into the object under test. The reflected pulse sequence in the time domain represents the discontinuities along the propagation direction, and the delay between the first pulse and each subsequent pulse indicates the distance between the detector and the reflecting surface. The distance can be calculated quantitatively when the refractive index of the materials are known.

The T-Ray 4000®was mounted on a scanning system in order to collect a 120x120 matrix of THz waveforms spaced by $1 \mathrm{~mm}$ (1mm/pixel). In analogy to ultrasound technology, THz matrix data can be shown as a $\mathrm{THz}$ area image (or C-scan) or a cross-sectional view (B-scan). The C-scan is similar to a photograph and presents a top-view image of the area that shows the intensity of the $\mathrm{THz}$ reflection at any desired subsurface depth. THz C-scans are obtained by extracting the intensity of the reflected pulses at a particular time delay. The B-scan is a cross-sectional view of one selected line of the scanned area. While a C-scan is useful to detect objects and the pattern of the bandaging, a B-scan shows the number and spacing of the bandage layers as well as the depth of an object.
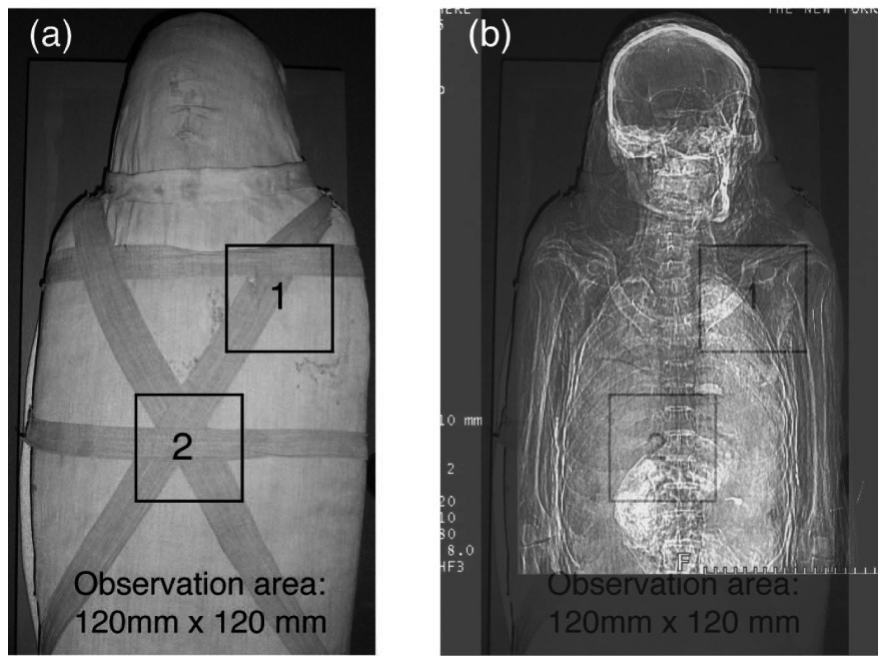

FIG. 1 Mummy of Kharushere (MMA 86.1.35, Funds from Various Donors, 1886) Kharushere lived during Egypt's Dynasty 22 (ca. $945-712$ B.C.). (a) Investigation areas 1 and 2, each measuring $120 \times 120 \mathrm{~mm}$, are highlighted in the figure, (b) Overlay of CT-scan and photographic image.

The potential and limitations of $\mathrm{THz}$ imaging as a tool to study mummy wrapping is illustrated by the data collected on the two areas highlighted in Fig. 1. These areas are relatively flat so that the incident angle of the $\mathrm{THz}$ waves was kept as close as 90 degree. The sequence and the number of bandage layers under the mummys shroud are evident in the C-scan and B-scan images, respectively, of Area 1 (Figs. 2(a) and $2(b))$. The depth probed however is quite limited. The (a)

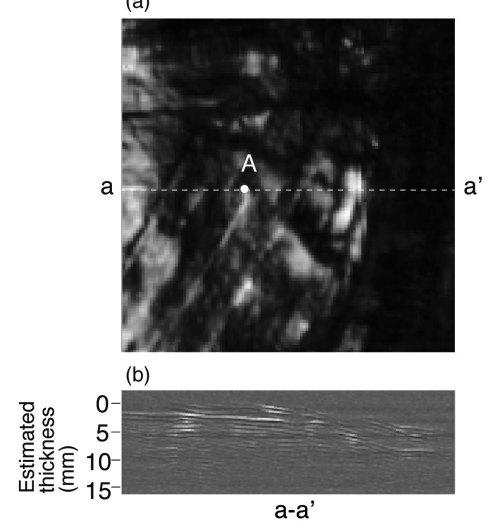

(c)

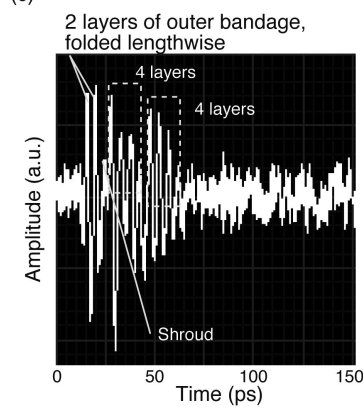

FIG. $2 \mathrm{THz}$ reflection images and an output signal waveform of the mummy of Kharushere, Area 1. (a) C-scan, (b) B-scan of the line $a-a^{\prime}$, (c) waveform extracted from the middle point $A$ of the line $a-a$ '.

waveform extracted from the middle point A of the line a-a (Fig. 2(c)) shows two strong reflections in correspondence of the diagonal strap that is circling the mummy from the proper left shoulder to the proper right hip, and then by two packets of four reflections each. The two reflections of the diagonal strap are explained by the fact that the strap is actually a bandage folded in half lengthwise and thus consisting of two fabric layers.

Here, it was noticed that the reflection signal power of the fabric layers was about 100 times smaller than that of glued paper layers in a previous study. [13]. This result suggests that $\mathrm{THz}$ waves pass through the outermost fabric, and are absorbed by the internal resin-fabric composite. Further basic research on spectra should help to estimate material properties.

The comparison with the CT-scan does not shed additional light on the eight reflections, as the bandages are even less clearly imaged by radiography. The sequence indicates four bandage layers followed by another set of four layers, with a gap in between. The total thickness of the eight fabric layers is approximately $9 \mathrm{~mm}$. The reflected signal decays quickly below approximately $12 \mathrm{~mm}$ from the surface.

The number of fabric layers observed is more than 10 in Area 2, as the B-scan in the c-c line shows (Fig. 3(c)). The B-scan along the $b-b^{\prime}$ line of Area 2 (Fig. 3(b)) shows several intense reflections coming from layers underneath the surface. A particularly intense reflection originates from a depth of about 20 $\mathrm{mm}$ from the surface (in correspondence of the point marked with B on Figs 3 and 4.

\section{COMPARISON WITH X-RAY CT}

Inspection of a CT section of the chest of the mummy taken approximately along the b-b line in Area 2 (Fig. 5) shows that the total thickness of the bandages over the chest of the mummy is approximately $60 \mathrm{~mm}$. To a depth of approximately $20-30 \mathrm{~mm}$ from the surface the bandages appear less radio-opaque in the CT-scan, while below this depth the radio-opacity of the bandages becomes more pronounced. This can be explained with the application of resins during the mummification process. As the bandages become satu- 
(a)

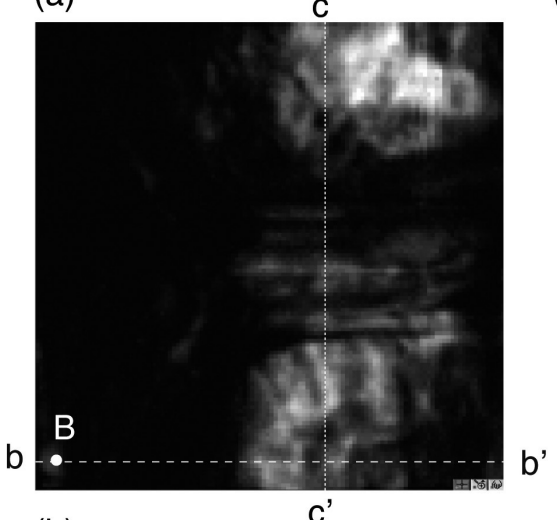

(b)

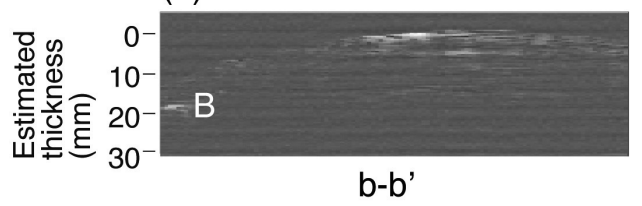

(c)

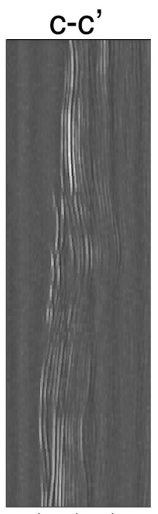

01020

Estimated thickness $(\mathrm{mm})$

FIG. 3 THz reflection images and an output signal waveform of the mummy of Kharushere, Area 2. (a) C-scan, (b) B-scan of the line b-b', (c) B-scan of the line $c-c^{\prime}$.

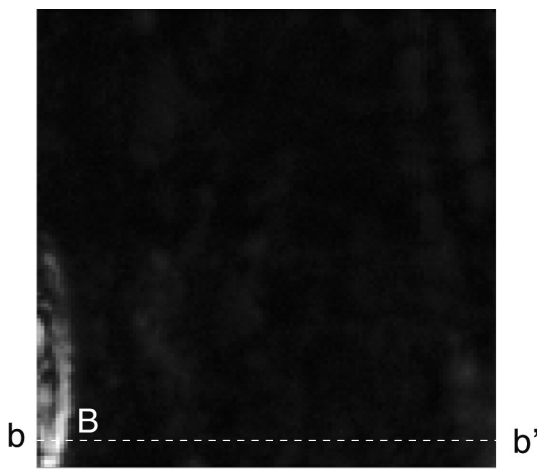

FIC. 4 THz reflection image of the C-scan of the mummy of Kharushere, Area 2, at the depth around $20 \mathrm{~mm}$ from the surface. A strong reflection is visible in the C-scan as well as in the B-scan of the $b-b^{\prime}$ line (B point in Fig. $3(b)$ ) at about $20 \mathrm{~mm}$ depth. This is possibly due to resin-impregnated bandage layers.

rated with resins (note the large amounts of pooled liquid congealed in the abdominal cavity), they become denser, and can be better seen in the radiographic image. Their refractive index also increases with the addition of the resin. The high intensity reflection observed in Fig. 4 probably originates at the interface between the resin-free outer bandages and the resin saturated bandages, approximately $80 \mathrm{~mm}$ to the viewers left of the spine (indicated by the arrow).

Comparison of the scans in Fig. 4 with the waveform in Fig. 2(c) highlights the difficulties in interpreting $\mathrm{THz}$ data on mummies. Reflections from bandages at depth below $10 \mathrm{~mm}$ are weaker and cannot be distinguished from the background. Where layers with higher reflectivity are encountered however (as in the case of the resin impregnated bandages), reflections can be observed from deeper into the wrappings.

With the increase of the power of $\mathrm{THz}$ waves, development of scanning system, as well as signal processing software, which are suitable for mummies in the future, we expect $\mathrm{THz}$ waves eventually to reach through all fabric layers and to the body.

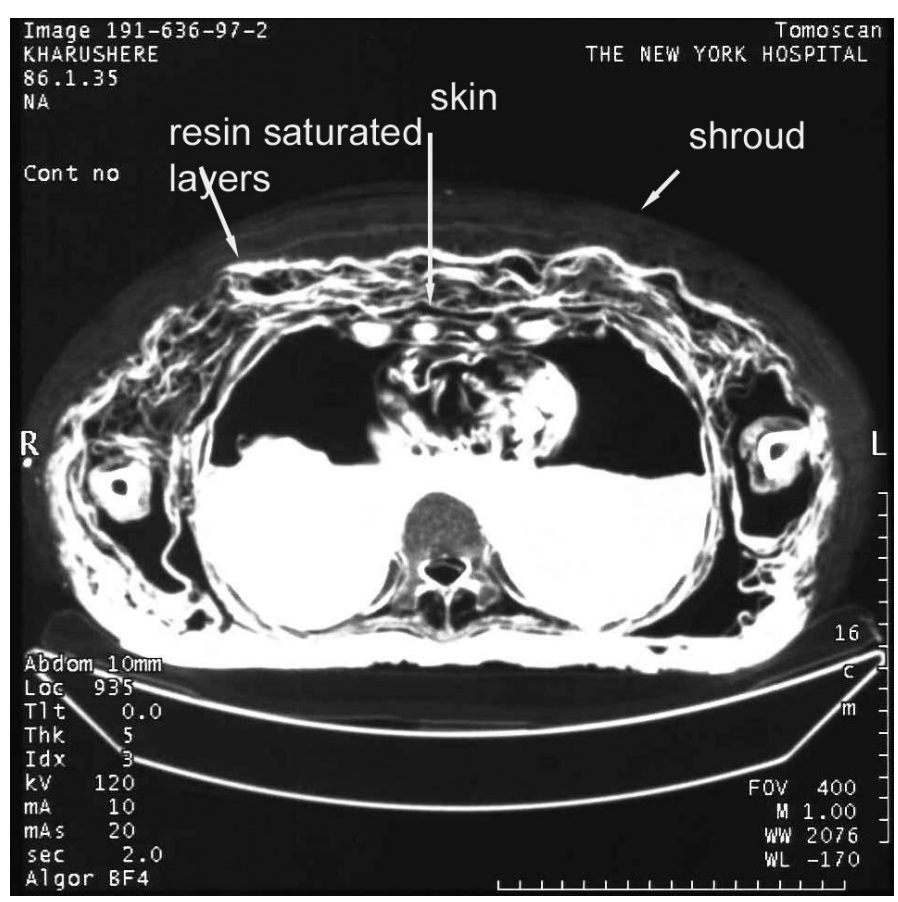

FIG. 5 CT scan of location no. 935, showing large amounts of congealed resins filling the abdominal cavity, resin saturated wrapping layers, and outer, resin-free, wrapping layers. Note that the CT-scan shows a section viewed from the bottom toward the head of the mummy. Therefore the mummyõs right side is on the left side of the image, as indicated by the $R$ and $L$ letters.

\section{CONCLUSIONS}

$\mathrm{THz}$ pulsed time domain reflection imaging is a promising technique for probing the fabric layers of Egyptian mummies. While the depth of penetration of $\mathrm{THz}$ waves is limited by comparison with radiographic methods, high contrast images close to the surface and detailed waveforms were obtained in the areas probed in this preliminary investigation. As CTscanning and $\mathrm{THz}$ instrumentation advance, we can expect improvements both in the ability of CT-scanning to image lighter materials (and studies conducted more recently than our 1997 CT scanning investigation of Karushere point in that direction [14]) and in the depth of penetration of $\mathrm{THz}$ waves. Although $\mathrm{THz}$ technology is not developed far enough yet to contribute to our knowledge of the textiles surrounding a mummy, it has the potential to become a useful tool in studying the fabric layers of mummies, since the technique of $\mathrm{THz}$ is to be expected to improve signicantly in the near future.

\section{ACKNOWLEDGEMENT}

The authors thank Dorothea Arnold, Lila Acheson Wallace Chairman, and Catharine Roehrig, Curator, of the Department of Egyptian Art, The Metropolitan Museum of Art, for their help in facilitating the experiments.

\section{References}

[1] Federico Cesarani, Maria Cristina Martina, Andrea Ferraris, Renato Grilletto, Rosa Boano, Elisa Fiore Marochetti, Anna Maria Donadoni and Giovanni Gandini, "Whole-Body Three-Dimensional Multidetector CT of 13 Egyptian Human Mummies", Am. J. Roentgenol., 180, 597-606 (2002). 
[2] Heidi Hoffman, William E. Torres, and Randy D. Ernst "Paleoradiology: Advanced CT in the Evaluation of Nine Egyptian Mummies", Radiographics, 22, 377-385 (2002).

[3] Rosalie. David Ed., Egyptian Mummies and Modern Science, (Cambridge University Press, Cambridge, 2008).

[4] Daniel Mittleman Ed., Sensing with terahertz radiation, (Springer, Berlin, 2003).

[5] Masayoshi Tonouchi, "Cutting edge terahertz technology", Nat. Photonics, 1 97-105, (2007).

[6] Kaori Fukunaga, Yuichi Ogawa, Shin'ichiro Hayashi and Iwao Hosako, "Terahertz Spectroscopy for Art Conservation", IEICE Electron. Expr.,4, 258-263 (2007).

[7] J.B. Jackson, M. Mourou, J.F. Whitaker, I.N. Duling III, S.L. Williamson, M. Menu and G.A. Mourou, "Terahertz imaging for non-destructive evaluation of mural paintings", Opt. Commun.,281, 527-532 (2008).

[8] J. Labaune, J. B. Jackson, S. Pages-Camagna, I. N. Duling, M. Menu and G. A. Mourou, "Papyrus imaging with Terahertz time domain spectroscopy", Appl. Phys. A-Mater., 100, 607-612 (2010).

[9] Kaori Fukunaga and Iwao Hosako, "Innovative non-invasive analysis techniques for cultural heritage using terahertz technology", C. R. Phys., 11, 519-526 (2010).

[10] L. Ohrström, A. Bitzer, M. Walther, F. J. Rźhli, "Technical Note: Terahertz Imaging of Ancient Mummies and Bone", Am. J. Phys. Anthropol., 142, 497-500 (2010).

[11] D.T. Mininberg, "The Museum's Mummies: an Inside View, Neurosurgery", 49, 192-199 (2001).

[12] I. N. Duling and David Zimdars, “Terahertz imaging: Revealing hidden defects", Nat. Photonics, 3, 630-632 (2009).

[13] N. Kamba, Y. Tsuchiya, A. Okimoto, K. Fukunaga, “Internal Structure Observation of a Japanese Panel Painted Screen by Terathertz Imaging Technique", in Proceeding 1oth International Conference on non-destructive investigations and microanalysis for the diagnostics and conservation of cultural and environmental heritage, NDT-41 (2011).

[14] J. Elias, C. Lupton, "The Role of Computed Axial Tomography in the Study of the Mummies of Akhmim", in Proceeding $V$ World Congress on Mummy Studies. (J. Biol. Res., 2005) pp. 34-38. 